\title{
Scaling pFET Hot-Electron Injection
}

\author{
Chris Duffy and Paul Hasler \\ Department of Electrical and Computer Engineering \\ Georgia Institute of Technology, Atlanta, GA 30332, USA \\ chris.duffy@ieee.org and phasler@ece.gatech.edu
}

This paper ${ }^{1}$ elaborates on a previously introduced [1] analytical model for hot-electron injection in p-channel MOSFET's. Hot-electron injection is frequently exploited to remove stored charge in floating-gate circuits. As illustrated in Figure 1, hole-induced impact ionization provides an otherwise empty conduction band with electrons (1). Although most of the electrons migrate to the bulk (2), under the proper conditions some inject through the gate oxide (3). It has been shown that impact ionization and hot-electron injection can be analytically solved using a self-consistent model derived from a spatially-varying Boltzmann transport equation [2],[3]. The primary mechanisms for altering the distribution function (and hence the collision operators for the BTE) are the aforementioned impact ionization and optical phonon absorption and emission.

We present data from devices fabricated on processes with minimum channel lengths of $2 \mu \mathrm{m}, 1 \mu \mathrm{m}$, $0.5 \mu \mathrm{m}, 0.35 \mu \mathrm{m}, 0.25 \mu \mathrm{m}, 0.18 \mu \mathrm{m}$, and $0.13 \mu \mathrm{m}$, modeling these devices analytically in each process. While we can derive the mean free length between phonon collisions, $\lambda$, using the well known energy of optical phonons in silicon [4], we must provide a theoretical model and experimental verification of the energy dependence of the mean free lengths between impact-ionization events in the conduction and valence bands, $L_{e^{-}}(E)$ and $L_{h^{+}}(E)$. Figure 2 verifies that the empirical results may be extrapolated to predict pFET behavior across all processes, including those below 100 $\mathrm{nm}$. Furthermore, we model data acquired at low temperature $(\sim 77 K)$, where the reduced phonon density gives insight into the effect of the phonon distribution on the charge carriers.

Figure 3 shows how the charge carriers' energies are altered through the drain-to-channel depletion region. The shape of these curves sheds light on the high-field carrier distribution as well as the effect of impact ionization on the mean kinetic energy of electrons and holes. Although Figure 3 implies newly generated carriers begin with little kinetic energy (i.e. they start at the band edge), it has been observed that this is not always the case [5]: such behavior can lead to higher average carrier energies, carrier multiplication, and a loop energy gain from the valence band to the conduction band and back again. Figure 4 further illuminates the role phonons play in altering the distribution function. The spreading of carriers through the depletion region is dependent upon the phonon density; i.e., the bell-shaped curve (insert) in Figure 4 remains narrower when there are fewer phonon emissions (which is true for processes with smaller device dimensions). The normalized impact-ionization and injection rates have been shown [1]: the injection rate is expressed as

$$
\frac{I_{\text {inj }}}{I_{\text {impact }}}=\int_{E_{\text {ox }}}^{\infty}\left(\exp \left(-\frac{\lambda}{z_{L}-z} \cdot\left(\frac{E-E_{1}(z)}{2 E_{R}}\right)^{2}\right)\right) \cdot\left(\exp \left(-\frac{1}{\left(q \mathcal{E} \lambda-E_{R}\right)} \int_{E=0}^{E} \frac{\lambda}{L(E)} d E\right)\right) d E
$$

\section{REFERENCES}

[1] Chris Duffy and Paul Hasler, "Modeling Hot-Electron Injection in pFET's," Journal of Computational Electronics, Vol. 2, No. 2, December 2003, pp. 317-322.

[2] E.M. Conwell, High Field Transport in Semiconductors, Academic Press, New York, 1967.

[3] Paul Hasler, Andreas Andreou, Chris Diorio, Bradley A. Minch, and Carver Mead, "Impact ionization and hotelectron injection derived consistently from Boltzmann transport," VLSI Design, 8(14), 455(1998).

[4] William Shockley, "Problems Related to p-n Junctions in Silicon," Solid-State Electronics, 1961, Vol. 2, No. 1, pp. $35-67$.

[5] J.D. Bude, B.E. Weir, P.J. Silverman, International Electron Devices Meeting '98 Technical Digest, December 1998, pp. $179-182$.

${ }^{1} \mathrm{~A}$ full journal publication of this work will be published in the Journal of Computational Electronics. 


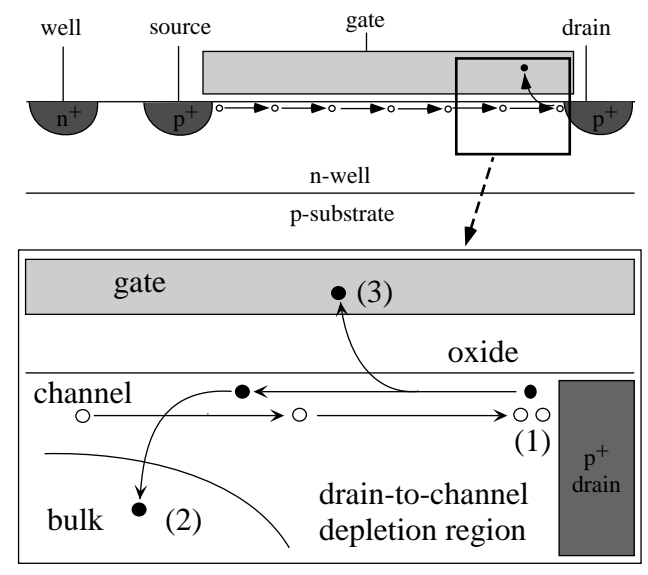

Figure 1. Hole-induced impact ionization and hotelectron injection in a pFET.
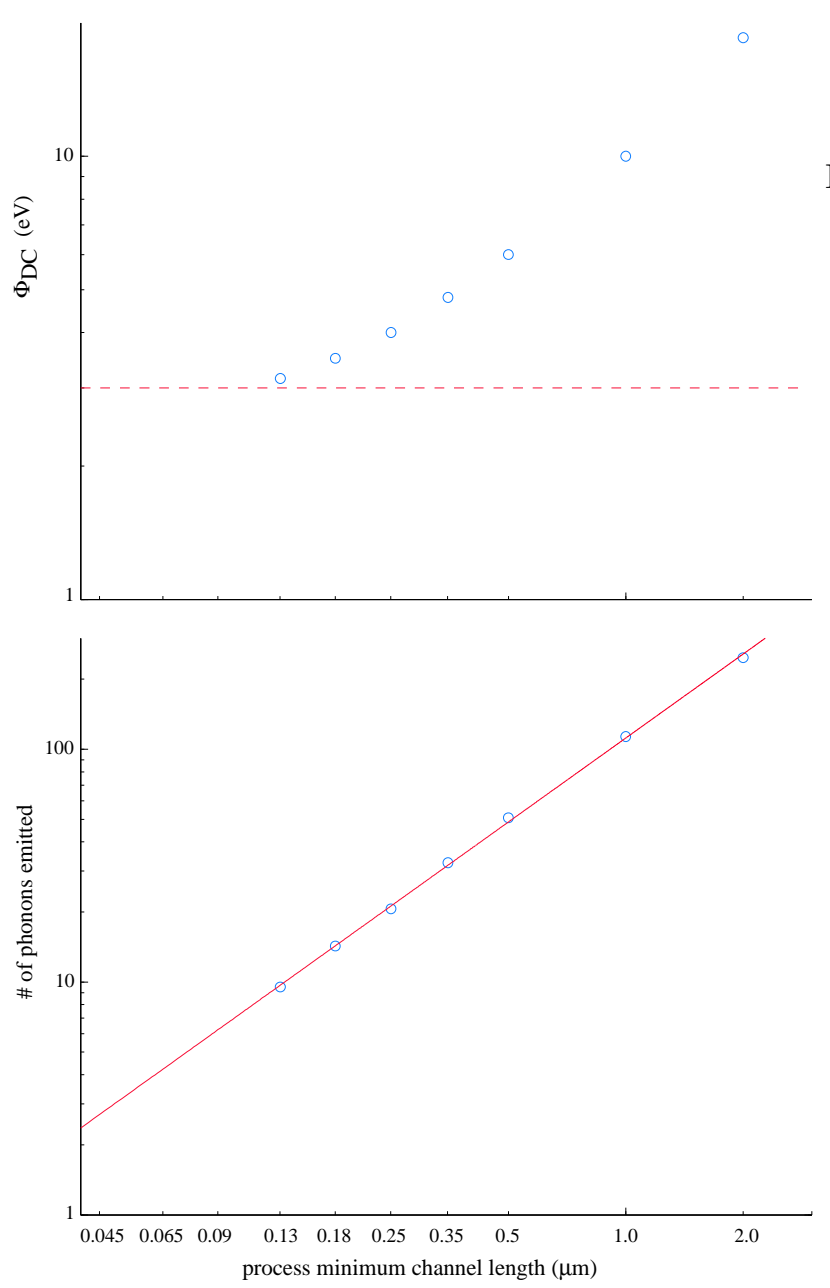

Figure 2. The necessary $\Phi_{D C}$ that achieves equivalent injection currents across processes asymptotically approaches the $\mathrm{SiO}_{2}$ barrier energy $(\sim 3 \mathrm{eV})$. The net number of phonons emitted from the average electron through the drain-to-channel depletion region decreases linearly with process reduction.

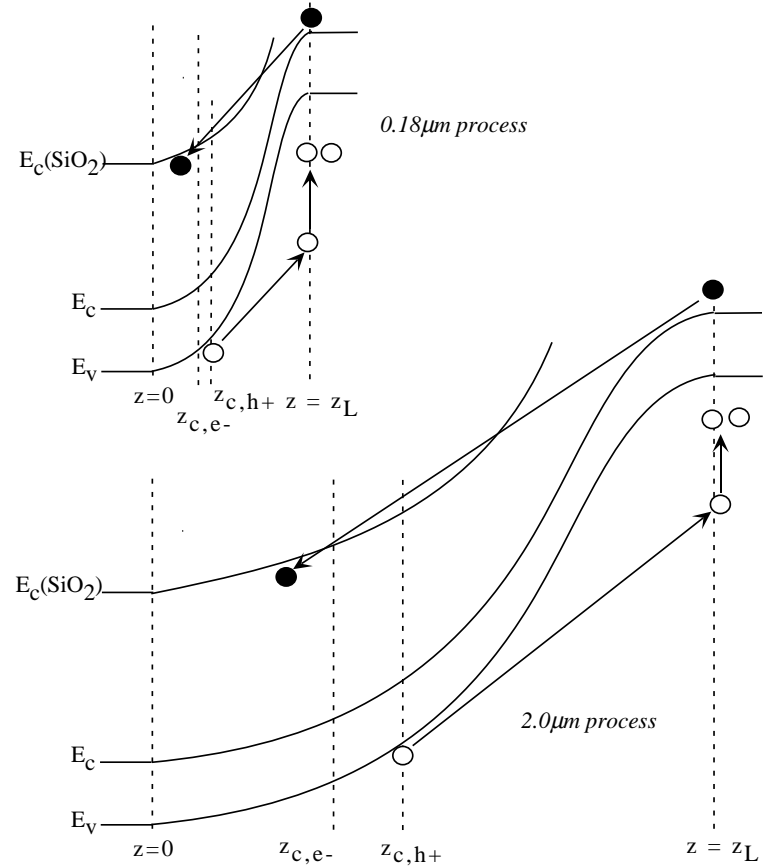

Figure 3. The electron-band diagram of the drainchannel depletion region of two pFET's in different processes exhibiting hot-electron injection. The critical $z$ for holes represents when the average hole leaves the conduction band, whereas $z_{c}$ for electrons designates where impact-ionization is most likely to occur in the conduction band (for electrons that have not been injected into the gate).
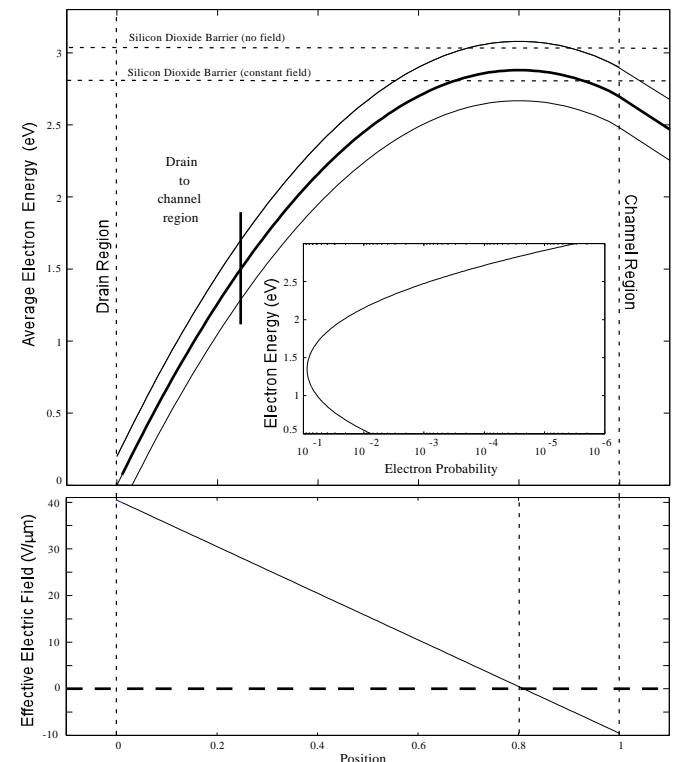

Figure 4. Model of hot-electron transport in the conduction band. Electrons are generated near the drain edge due to impact ionization.. Lines for zero and constant field are given to show what energy is necessary and how the barrier lowering changes this problem. 